\title{
Study of Nanoparticles Interaction with Biological Tissues Using Comparative Optical-Spectroscopic Methods.
}

\author{
E.V.Perevedentseva ${ }^{1,4}$, A.V.Karmenyan ${ }^{1}$,Y.C. Lin $^{1}$, Ashek-I-Ahmed ${ }^{1}$, N.Ali $^{2}$, M. Kinnunen ${ }^{3}$, O. Bibikova ${ }^{2,3,}$ \\ I. Skovorodkin ${ }^{2}$, S. Vainio ${ }^{2}$, C.L. Cheng ${ }^{1}$ \\ ${ }^{1}$ Department of Physics, National Dong Hwa University, Hualien, Taiwan \\ ${ }^{2}$ Biocenter Oulu, University of Oulu, Oulu, Finland \\ ${ }^{3}$ Opto-Electronics and Measurement Techniques Research Unit, Faculty of Information Technology and Electrical Engineering, \\ University of Oulu, Oulu, Finland \\ ${ }^{4}$ P.N. Lebedev Physics Institute Rus. Acad. Sci. Moscow Russia \\ elena@gms.ndhu.edu.tw, clcheng@gms.ndhu.edu.tw
}

\begin{abstract}
Recent development of nanoparticles bio-medical applications is determined by perspectives of their use for multimodal bio-imaging and sensing. Informative and noninvasive optical-spectroscopic methods are designed for the detection and analysis of the NP interaction with target biological systems. Presented work is focused on study of nanoparticles interaction with biological tissues combining complimentary methods to obtain versatile optical-spectroscopic information.
\end{abstract}

Keywords - nanoparticles bio-medical applications; OCT; confocal microscopy; 2-photon microscopy; fluorescence lifetime analysis; FLIM; Raman spectroscopy

Recent intense development of the nanoparticles (NP) applications in the biology and medicine is in high degree determined by new facilities opened by their multifunctional use [1]. The NP multifunctional bio-medical applications include first of all multimodal imaging with the NP like bionano-probes $[1,2]$; that implies application and development of various methods of detection of NP interaction with target for obtaining versatile and complementary information. It is important that an imaging can be also combined with simultaneous drug delivery and treatment [1]. On the other hand combining different optical-spectroscopic methods allow studies of mechanisms of processes in living systems involving NP like a probes and discussion of the nanosafety problems.

To study the NP interaction with biological systems and their models we are combining number of opticalspectroscopic methods and discuss their optimal applicability and informativeness. Previously, to explore the effects of diamond nanoparticles (nanodiamonds, ND) with different physicochemical properties on the developing zebrafish embryo, optimize visualization in the embryo model, and develop methods and criteria of the toxicity estimation we combined confocal microscopy, 2-photon microscopy and lifetime imaging [3]. To study ND interaction with tissues of blood circulation system on the human and rat models, the influence of ND of various surface properties on the blood system functioning a confocal microscopy has been combined with Raman spectroscopy and Raman mapping [4].
Presented work is focused on study of interaction of different nanoparticles (NDs of different size and surface properties; gold nanostars) with biological tissues, using the models of mice skin. The optical non-invasive imaging methods for both macro- and micro- imaging and visualization are combined: optical coherence tomography (OCT), fluorescence confocal imaging and fluorescence lifetime imaging (FLIM). The results obtained using three complimentary methods allow to observe comprehensively the NP penetration and distribution in skin, and to discuss penetration pathways for studied models and extension of the obtained information for other model systems.

Applications of several complimentary methods of imaging and spectroscopic analysis allow optimizing the experiments taking into account the NP's and sample's properties and the scientific problem in hand. Optimal combination of the methods also opens ways for NP applications like advanced marker or contrast agent for bio-imaging and for bio-sensing.

\section{ACKNOWLEDGMENT}

The authors appreciate the financial support of this research by Ministry of Science and Technology of Taiwan, Grant No. MOST 103-2112-M-259-001-MY3 and the Academy of Finland (grant No. 297542).

\section{REFERENCES}

[1] D.E. Lee, H. Koo, I.C. Sun, J.H. Ryu, K. Kim K, I.C. Kwon. "Multifunctional nanoparticles for multimodal imaging and theragnosis, " Chem. Soc. Rev. vol. 41(7), pp. 2656-2672, April 2012

[2] W. W.- W. Hsiao, Y. Y. Hui, P.- C. Tsai, H.- C. Chang. "Fluorescent Nanodiamond: A Versatile Tool for Long-Term Cell Tracking, SuperResolution Imaging, and Nanoscale Temperature Sensing," Acc. Chem. Res., vol. 49(3), pp. 400-407, March 2016.

[3] Y.C. Lin, K.T. Wu, Z.R. Lin, E. Perevedentseva, A. Karmenyan, M.D. Lin, C.L. Cheng. "Nanodiamond for biolabelling and toxicity evaluationin the zebrafish embryo in vivo." J Biophotonics, vol. 9(8), pp. 827-836, Aprel 2016.

[4] L.W. Tsai, Y.C. Lin, E. Perevedentseva, A. Lugovtsov, A. Priezzhev, C.L. Cheng. Nanodiamond for medical applications: interaction with blood in vitro and in vivo. Int $\mathrm{J}$ Mol Sci in a special issue: Inorganic Nanostractures in Biological Systems, vol. 17(7), p. 1111, July 2016. 